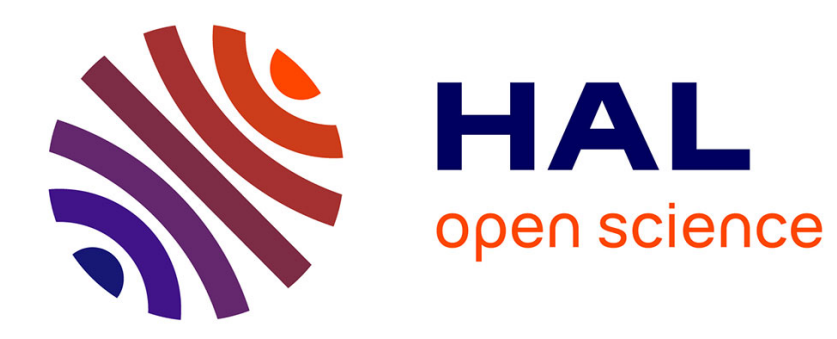

\title{
La codification du droit international privé
}

Hervé Lecuyer

\section{- To cite this version:}

Hervé Lecuyer. La codification du droit international privé. Revue juridique de l'Océan Indien, 2004, 04, pp.63-76. hal-02541645

\section{HAL Id: hal-02541645 \\ https://hal.univ-reunion.fr/hal-02541645}

Submitted on 14 Apr 2020

HAL is a multi-disciplinary open access archive for the deposit and dissemination of scientific research documents, whether they are published or not. The documents may come from teaching and research institutions in France or abroad, or from public or private research centers.
L'archive ouverte pluridisciplinaire HAL, est destinée au dépôt et à la diffusion de documents scientifiques de niveau recherche, publiés ou non, émanant des établissements d'enseignement et de recherche français ou étrangers, des laboratoires publics ou privés. 


\title{
LA CODIFICATION DU DROIT INTERNATIONAL PRIVE
}

\author{
Par Hervé LECUYER \\ Professeur à l'Université Panthéon-Assas (Paris 2)
}

1. Si Bentham avait vaincu, imposant à tous son code universel et intégral, le Pannomion ${ }^{1}$, la question de la codification du droit international privé ne se poserait poserait pas, faute d'objet. Il ne triompha pas (qui eut pu douter de son échec ?). La question demeure ouverte. Elle n'agite pas seulement les spécialistes du droit international privé. La codification du droit international public est d'actualité. L'article 13 par 1 (a) de la Charte des Nations Unies donne, à cette fin, mandat à l'Assemblée générale de «provoquer des études et de faire des recommandations en vue d'encourager le développement progressif du droit international et sa codification ». Cette codification là, recherchée et encouragée, n'a sans doute pas la consistance que les juristes privatistes lui reconnaissent. La communauté internationale ne dispose d'aucun organe législatif; la codification s'entend dès lors d'une «transcription de règles coutumières en un corps de règles écrites » ${ }^{2}$ par le canal des Traités. La codification pourrait se résumer, dans cet ordre, à la création de règles conventionnelles reflétant les règles coutumières.

2. L'idée même d'une codification du droit international privé est récente ${ }^{3}$. Il Il manquait, il est vrai, à la matière, la dimension écrite, prémisse ou accompagnement de toute codification. Abandonnée à la jurisprudence et à la doctrine (jouant ici un rôle prépondérant), elle tolérait exceptionnellement l'intrusion du législateur, national ou international. Il fallut ainsi attendre 1854 pour qu'une première codification, systématique et complète du droit international privé vit le jour, dans le Canton de Zurich. Auparavant, quelques règles éparses furent retrouvées, en Bavière ${ }^{4}$ ou en Prusse ${ }^{5}$, dans des codes dont l'objet dépassait le droit droit international privé et qui n'accueillait souvent ce dernier que de manière partielle ${ }^{6}$. Le précédent Zurichois encouragea sans doute à de nouvelles expériences, expériences, au XIXe siècle ${ }^{7}$, mais l'élan ne fut réellement pris qu'au XXe siècle, animant l'Europe ${ }^{1}$ voire le monde entier ${ }^{2}$.

\footnotetext{
${ }^{1}$ Sur lequel, v. R. Cabrillac, Les codifications, PUF, Droit, éthique, société, p. 37.

${ }^{2}$ R. Cabrillac, préc., p. 43.

${ }^{3}$ B. Nolde, «La codification du droit international privé », Académie dr. int. Rec. des cours, tome 55 (1936-I), p. 299 et s.

${ }^{4}$ En 1756.

${ }^{5}$ En 1794.

${ }^{6}$ E. Jayme, «Considérations historiques et actuelles sur la codification du droit international privé », Académie dr. int. Rec. des cours, tome 177 (1982-IV), p. 9.

${ }^{7}$ Le royaume de Saxe codifia son droit international privé, dans son code civil, en 1863 ; Le code civil italien y consacra, en 1865, ses dispositions préliminaires. V. E. Glasson, « La codification en Europe au
} 
3. L'essor des codifications nationales s'est accompagné d'un imposant mouvement de codification internationale, compris comme une expansion du droit conventionnel $^{3}$, sous l'auspice des instances européennes, de l'ONU ou de la Conférence de la Haye ${ }^{4}$.

4. La France semble, jusqu'à présent, s'être tenue à l'écart de ce mouvement d'ampleur ${ }^{5}$. Le constat peut rapidement être dressé : le droit international privé français ne fait l'objet d'aucune codification globale, ne repose même pas sur un corps ordonné de règles posées par le législateur. 1804 ne fut sans doute pas une date propice à l'éclosion d'un code et le laconisme du Code civil en la matière ne peut qu'être souligné. Il est vrai que, selon la célèbre formule de Lainé, « le Code civil fut rédigé en un temps où les conflits s'élevaient entre les armées des peuples plutôt qu'entre leurs lois ${ }^{6}$. En outre, les relations privées internationales ne concernaient alors qu'une élite, les rédacteurs du code civil ne disposaient, sur ces matières, d'aucuns travaux équivalents à ceux de Domat ou Pothier, pour le droit civil, enfin les doctrines existantes révélaient - déjà - une profonde diversité, voire de réels antagonismes. Comment, pour le législateur, songer à une synthèse ?

5. Les tentatives ultérieures de codification furent nombreuses. Aucune n'aboutit. On doit à Niboyet un premier projet, en $1954^{7}$. Comprenant 150 articles, l'ambitieux projet, très marqué par les idées de son inspirateur, reçut un accueil réservé par le Comité français de Droit international privé, ce qui le condamnait ${ }^{8}$. Le deuxième projet fut rédigé par Batiffol. Son destin se limita à son adoption, le $1^{\text {er }}$ juillet 1959, par la Commission de réforme du Code civil. Un troisième projet, enfin, fut établi par une Commission, présidée par M. Jean Foyer, siégeant à la

XIXe siècle», Rev. pol. et parl. p. 201 et 402, 1895, p. 198 ; D. Bureau, «La codification du droit international privé », in La codification, Dalloz, Thèmes et commentaires, 1996, p. 119 et s., spéc. p. 120.

${ }^{1}$ Connurent une codification (isolée ou intégrée à une œuvre plus imposante) : la Tchécoslovaquie, la Pologne, la RDA, la Hongrie, la Turquie, le Portugal, l'Espagne, l'Autriche, la RFA, la Suisse et l'Italie. $\mathrm{V}$. D. Bureau, préc. et les très nombreuses références doctrinales comprises dans sa contribution; E. Vassilakakis, Orientations méthodologiques dans les codifications récentes du droit international privé en Europe, LGDJ 1987, Préf. P. Lagarde.

${ }^{2}$ F. Rigaux, «La méthode des conflits de lois dans les codifications et projets de codification de la dernière décennie », Rev. crit. DIP 1985, p. 1.

${ }^{3}$ Le sens donné à la codification est ici encore différent de celui généralement retenu par les privatistes.

${ }^{4}$ D. Bureau, op. cit., p. 123 ; B. Oppetit, «le droit international privé, Droit savant », Académie dr. int. Rec. des cours, tome 234 (1992-III), p. 411 et s.

${ }^{5}$ Y. Loussouarn, «Les vicissitudes de la codification en droit international privé français », Mélanges Droz, p. 191.

${ }^{6}$ A. Lainé, Etude sur le titre préliminaire du projet de révision du code civil belge, Bulletin de la Société de Législation comparée, 1890 , p. 7.

${ }^{7}$ Avant-projet du code civil présenté à M. le Garde des Sceaux, ministre de la Justice, par la Commission de réforme du Code civil, Première partie, Livre préliminaire, Publications du Ministère de la Justice, 1954.

${ }^{8}$ V. J.P. Niboyet, « Le code civil en préparation et les règles de solution des conflits de lois », Trav. Com. fr. DIP 1945-1946, p. 13 ; Y. Loussouarn, « Le projet de code de droit international privé et le colloque relatif à la codification du droit international privé », RTD com. 1955, p. 800. 
Chancellerie, du 10 décembre 1965 au 2 janvier $1967^{1}$. Ce texte, destiné à compléter le Code civil en matière de droit international privé, et comportant 37 articles, fut aussi fraîchement reçu par la doctrine. Il ne connut pas de lendemain. Une explication peut-elle être donnée à ces échecs successifs? La raison la plus apparente est d'ordre conjoncturel. L'abandon des deux premiers projets peut s'expliquer par l'enterrement du projet général de réforme du Code civil ; le départ du Garde des Sceaux causa vraisemblablement l'échec du dernier. Sans doute convient-il de ne pas se limiter aux raisons apparentes, même si elles ont pu avoir leur part dans les revers subis par les projets successifs. Substantiellement, l'explication peut être trouvée dans les réserves doctrinales émises à l'encontre de chacun d'eux. Est ainsi révélée, sous ce jour, le rôle éminent joué par la doctrine internationaliste; nous le retrouverons ultérieurement.

6. Ce vide de code n'est pas absolu, en droit français, et l'on peut recenser les traces de codification partielle du droit international privé ${ }^{2}$. Le droit de la nationalité, nationalité, après des allers-retours ${ }^{3}$, se trouve aujourd'hui campé aux articles 17 à 33-2 du Code civil ${ }^{4}$. Les conflits de juridictions ont notamment pour socle les articles 14 et 15 du Code civil ainsi que les articles 42 et s. du Nouveau Code de procédure civile, si l'on admet, avec une jurisprudence, ${ }^{5}$ que la compétence internationale des juridictions françaises se détermine par extension des règles de compétence territoriale interne. Participent encore de cette codification partielle les dispositions relatives aux conflits de lois en matière de filiation ${ }^{6}$ et de divorce ${ }^{7}$ et quelques autres encore. Mais tout ceci est au mieux constitutif de parcelles de droit international privé codifié, non d'une codification du droit international privé. Dominique Bureau l'a parfaitement souligné : «l'ampleur des réalisations, la cohérence d'ensemble, la systématicité que postulent l'idée de codification sont ici largement absentes ».

7. La France n'est, de plus, liée par aucune codification internationale globale du droit international. On ne peut négliger l'ampleur des réalisations effectuées à la Haye. Au-delà, des traités d'inspiration européenne ont également modifié

\footnotetext{
${ }^{1}$ Jacques Foyer, Le nouvel avant-projet de réforme du droit international privé français, Clunet, 1971, p. 31 .

${ }^{2}$ M. Simon-Depitre, Codification et règles écrites dans le droit français des conflits des lois, in Liber Memorialis François Laurent, Bruxelles, Story-Scientia, 1989, p. 1039.

${ }^{3}$ P. Guiho, La nouvelle révision du code de la nationalité...et son abolition, D. 1994, chron. p. 1.

${ }^{4}$ Pour une approche critique, v. H. Muir Watt, La codification en droit international privé, Droits, $\mathrm{n}^{\circ} 27$, 1998 , p. 150.

${ }^{5}$ Cass. civ. 30 oct. 1962 (Scheffel), Rev. crit. DIP 1963, p. 387, note Francescakis ; D. 1963, p. 109, note G. Holleaux.

${ }^{6} \mathrm{H}$. Battifol et P. Lagarde, L'improvisation de nouvelles règles de conflit de lois en matière de filiation, Rev. crit. DIP 1972, p. 1 ; J. Foyer et M. Simon-Depitre, La loi du 3 janvier 1972 et le droit international privé, JCP 1973, I, 2566.

${ }^{7} \mathrm{Ph}$. Francescakis, Le suprenant article 310 nouveau du code civil sur le divorce international, Clunet 1976, p. 826.
} 
sensiblement le visage de la matière sur des questions essentielles ${ }^{1}$. Ce droit conventionnel répond-il aux canons de toute codification? Au mieux, il pourrait s'agir d'une codification partielle. Seules quelques matières, voire quelques questions sont ainsi réglementées. Au-delà, la codification poursuit un double objectif de cohérence et de simplification. Les conventions internationales n'y parviennent pas $^{2}$. La compatibilité même de ces instruments conventionnels avec la notion de codification est très discutable ; la doctrine, semble-t-il majoritaire, entend d'ailleurs réserver le terme à l'hypothèse d'une codification nationale, préférant parler d'unification pour ce qui relève des dispositions comprises dans les conventions internationales ${ }^{3}$.

8. La codification s'entend de l'opération d'élaboration d'un code, incluant le processus et son résultat, le code lui-même. Pour le droit international privé, il y a eu processus avorté, et il n'y a pas de résultat. La codification n'existant pas, de lege lata, la question est nécessairement posée de la perspective d'une codification future. Une telle codification est-elle possible ? Et si l'on répond par l'affirmative à cette première question, encore conviendra-t-il d'affronter une seconde : une telle codification verra-t-elle le jour?

\section{I/ LA FAISABILITE D’UNE CODIFICATION DU DROIT INTERNATIONAL PRIVE}

9. Les obstacles traditionnellement opposés à la perspective d'une codification peuvent être levés. En revanche, l'entreprise de codification demeurera inéluctablement soumise à diverses contraintes.

\section{A/ Les obstacles}

Le droit international privé rencontre, sur le chemin de la codification, des obstacles inhérents à toute entreprise de codification et d'autres qui pourraient lui être propres.

\section{$1 \%$ Les obstacles inhérents à toute entreprise de codification}

10. Tout code appelle un géniteur. Le problème est posé de distinguer un homme ou un groupement d'individus qui auraient la compétence requise pour mener à bien une codification du droit international privé. La question de la compétence est posée de longue date. On dit ainsi que les rédacteurs du Code civil, confrontés à cette matière, auraient été conscients de ce qu'ils ne maîtrisaient qu'imparfaitement les réponses à des problèmes complexes et préférèrent dès lors,

\footnotetext{
${ }^{1}$ Sur les conflits de juridictions : on songe bien sûr à la Convention de Bruxelles de 1968, récupérée par un Règlement communautaire. Sur les conflits de lois en matière d'obligations contractuelles, c'est à la Convention de Rome, en date du 19 avril 1980, qu'il convient de se référer. Faut-il encore rappeler l'inquiétante propension du droit communautaire à légiférer en la matière par voie de directive ? Procédé malheureux et dangereux. V. P. Lagarde, «Heurs et malheurs de la protection internationale du consommateur dans l'Union européenne », Mélanges J. Ghestin, LGDJ, 2001, p. 511.

${ }^{2}$ Y. Lequette, «De l'utilitarisme dans le droit international privé conventionnel de la famille », in Mélanges en l'honneur de Y. Loussouarn, Dalloz, 1994, p. 245.

${ }^{3}$ D. Bureau, op. cit., $\mathrm{n}^{\circ} 28$.
} 
se retrancher dans une posture d'abstention, laissant libre champ à la jurisprudence. Le Garde des Sceaux, Jean Foyer, constatait, au milieu des années 1970, que l'hémicycle des Assemblées ne serait pas un théâtre prédestiné pour entretenir des discussions sur le droit international privé. Il proposait alors, pour y remédier, de faire du recyclage, ou même simplement du «cyclage » de députés en cette matière ${ }^{1}$. Le propos conserverait, aujourd'hui encore, toute sa force.

11. Mais une telle quête d'un géniteur compétent est générale et intéresse toutes les branches du droit. Il n'est que de suivre les travaux préparatoires ayant précédé l'élaboration des lois récentes en droit civil ou en droit commercial pour en être convaincu. L'obstacle n'est cependant pas dirimant. Il est loisible au législateur, animé du complexe de Socrate, de s'en remettre à un ou des sachants pour l'élaboration de l'avant-projet. Le procédé est éprouvé; les résultats en sont excellents ${ }^{2}$. On ne soulignera jamais assez les apports de la doctrine à l'entreprise de législation. L'association pourrait, ici encore, être envisagée.

\section{$2 \%$ Les obstacles inhérents au particularisme de la matière codifiée}

12. Les obstacles liés au particularisme de la matière codifiée paraissent nombreux. On peut les regrouper en deux catégories, selon qu'ils ont trait aux caractères du droit international privé ou aux sources de ce dernier.

\section{a) Obstacles inhérents aux caractères du droit international privé}

Le droit international privé serait jeune, complexe et souple, plastique, adaptable, autant de caractères contrariant l'idée de codification.

13. «La vie d'un corps de règles juridiques, à l'instar de la vie humaine, passerait de l'enfance à la jeunesse puis à la maturité et enfin à la vieillesse. La codification serait souhaitable quand la règle juridique atteint une certaine maturité $»^{3}$. Or, l'idée demeure partagée que le droit international privé demeure une matière relativement neuve, comprenant des espaces sinon en friche, du moins imparfaitement explorés. L'argument fut opposé à la codification de la matière à l'époque du Code civil ; il le fut encore au projet Niboyet : la matière ne possédait alors pas le degré de maturité nécessaire pour s'offrir à la codification, disait-on. L'argument sonne faux, et a sans doute permis d'en masquer d'autres, moins avouables. Il est difficile de ne pas adhérer à la proposition suivante, valable, d'ailleurs pour toutes matières auxquelles il est fait grief de leur jeunesse : soit certaines solutions peuvent être tenues pour acquises, et la codification permet de les rappeler fermement; soit d'autres demeurent douteuses, et l'on pourrait alors se demander s'il n'est pas précisément souhaitable de faire cesser l'incertitude ${ }^{4}$.

\footnotetext{
${ }^{1}$ J. Foyer, Trav. Com. fr. DIP 1975-1977, p. 194.

${ }^{2}$ Songeons à la préparation de la réforme du 3 décembre 2001, relative aux droits du conjoint survivant et des enfants adultérins, intervenue dans une matière qui, à ce que l'on en sache, n'est guère moins complexe que celle du droit international privé.

${ }^{3}$ R. Cabrillac, op. cit., p. 257 ; Fr. Terré, « Les problèmes de la codification à la lumière des expériences et des situations actuelles », in Etudes de droit contemporain, éd. Cujas, 1962, p. 175.

${ }^{4}$ D. Bureau, op. cit., $\mathrm{n}^{\circ} 34$, p. 133.
} 
L'argument tiré de la jeunesse d'une matière, enfin, paralyserait toute codification. En effet, le progrès scientifique et l'évolution des idées sont des choses auxquelles on ne peut jamais assigner un terme ${ }^{1}$. Une matière est toujours jeune, si l'on ne considère un tel qualificatif comme un non sens.

14. Nul ne niera la complexité du droit international privé. La matière est rendue difficile par son objet: les rapports de droit qu'il régit s'inscrivent dans le cadre de la vie internationale, élément certain de complexité ${ }^{2}$. Elle l'est encore par ses mécanismes. L'argument ne porte cependant pas. Il encourage, au contraire, à la codification. Le Code doit servir à exprimer des réalités complexes.

15. Pour beaucoup, enfin, la souplesse et l'adaptabilité du droit international privé encouragent à dénoncer le principe même de la codification, à critiquer la rigidité qu'elle engendrerait inévitablement. S'ils s'accordent sur le fait que la codification favoriserait sans doute la sécurité du droit et accroîtrait la prévisibilité des solutions, ils déplorent, à l'avance, la rigidité des solutions judiciaire que coûterait le surcroît de prévisibilité. La perspective n'est sans doute pas aussi terne. La rigidité n'est pas inéluctablement liée au phénomène de la codification et l'on peut toujours prévenir ce danger par l'utilisation de formules souples pour ne pas entraver la jurisprudence. Qui nierait que cette dernière n'a guère été bridée par le Code civil ? Entre la recherche d'une solution adaptée au cas particulier et le respect de la prévisibilité générale des solutions, la marge de manœuvre est étroite, mais elle existe.

Aucun des arguments, tirés des caractères de la matière, et généralement invoqués pour s'opposer à la codification du droit international privé n'est déterminant.

\section{b) Obstacles inhérents aux sources du droit international privé}

16. La matière est essentiellement jurisprudentielle ${ }^{3}$, ce qui fait, pour certains, son charme ${ }^{4}$. Cela ne constitue pourtant pas un obstacle insurmontable à la codification. Le droit comparé, et notamment l'exemple des pays de Common Law convainquent qu'une codification destinée à recueillir et organiser des règles d'origine jurisprudentielle est possible ${ }^{5}$. La réception du droit non écrit par le droit

\footnotetext{
${ }^{1}$ A. Ferrer-Correira, Les problèmes de codification en droit international privé, Académie dr. int. Rec. des cours, tome 145 (1975-II), p. 58.

${ }^{2}$ Y. Loussouarn et P. Bourel, Droit international privé, Dalloz, $7^{\mathrm{e}}$ éd., $\mathrm{n}^{\circ} 1$; adde. P. Mayer : «la caractère particulièrement complexe des problèmes appelle une conception des règles à partir de l'examen des cas concrets. Faute de ce guide, l'esprit humain, quelle que soit sa puissance d'abstraction, risque d'errer », in «Les réactions de la doctrine à la création du droit par les juges en droit international privé », Trav. Ass. Capitant, t. XXXI, Economica, 1982, p. 388.

${ }^{3}$ Ce fut vrai. Ce l'est moins.

${ }^{4}$ M. Ancel, Trav. Com. fr. DIP, 1945-46, p. 38.

${ }^{5}$ O. Moréteau, Droit anglais des affaires, Dalloz, 2000, $\mathrm{n}^{\circ} 718$.
} 
écrit ne doit pas effrayer dans un pays où l'habitude se prit de rédiger les coutumes ${ }^{1}$.

17. Le principal obstacle tenant aux sources du Droit international privé est ailleurs et tient dans le développement du droit conventionnel, le pullulement des sources internationales du droit international. On l'a dit, «la détermination des solutions de conflits de lois se voit désormais confiée à la compétence d'une pluralité d'auteurs normatifs : dès lors, l'unité de la source de réglementation est définitivement rompue. De fait, ce n'est pas le moindre paradoxe de la codification que d'avoir confié l'élaboration d'une matière aussi complexe non seulement à des autorités distinctes, mais surtout à des ordres juridiques différents : il ne s'agit pas ici tant de dénoncer le rôle dévolu à l'ordre international dans la détermination des solutions de conflits de lois, mais plutôt de s'interroger sur le bien fondé de la compétence concurrente qui est désormais reconnue aux autorités nationales et aux instances internationales pour le traitement de questions identiques $»^{2}$.

18. La situation est encore compliquée par le contenu et l'ambition très divers des conventions internationales. En matière de conflits de lois, certaines se bornent à unifier la solution du conflit de lois dans les pays signataires. D'autres tendent, en revanche, au-delà de l'unification de la règle de conflit, à écarter la diversité même des règles de fond en élaborant une loi uniforme régissant au fond les relations internationales ${ }^{3}$. Ce qui emporte superposition de cette loi internationale et des lois internes (qui continuent à régir les rapports internes). Pour en sortir, il conviendrait d'unifier complètement les lois des Etats signataires, aussi bien dans les relations internes qu'internationales, ou bien, pour les Conventions, réduire leurs ambitions.

19. La codification impose, pour pouvoir être réalisée, une unité, au moins relative, des sources normatives. Leur éclatement et leur multiplication, aux plans interne comme international, semble rendre l'entreprise inutile, voire impossible. L'expansion du droit conventionnel sème le doute quant à la pertinence d'une codification nationale. L'argument porte, mais n'est cependant pas pleinement convaincant. La codification nationale n'est pas interdite, mais son périmètre sera affecté par le mouvement de développement des sources internationales.

\section{B/ Les contraintes}

\footnotetext{
${ }^{1}$ V. le discours final de Portalis, exposant les motifs du projet de loi sur la réunion des lois civiles en un seul corps de lois, sous le titre de Code civil des Français, publié au JCP éd. G. 2004, I, 122 : «Dans les temps moins reculés on crut avoir fait un grand pas vers le bien quand on eut l'idée et le courage, je ne dis pas de réformer les anciennes coutumes, mais d'ordonner qu'elles seraient rédigées par écrit. Cette époque est célèbre dans l'histoire de notre ancienne législation; car des coutumes écrites, quoique d'ailleurs plus ou moins barbares, plus ou moins sages dans leurs dispositions, firent disparaître les inconvénients attachés à des conditions incertaines et variables. Les affaires de la vie prirent un cours plus fixe et plus régulier ; il y eut plus de sûreté dans l'ordre des successions, dans les propriétés privées et dans toutes les transactions sociales ».

${ }^{2}$ L. Gannagé, La hiérarchie des normes et les méthodes du droit international privé, Etude de droit international privé de la famille, LGDJ, Bibl. dr. privé, tome 353, préf. Y. Lequette, ${ }^{\circ} 4$.

${ }^{3}$ P. Chauveau, «Les conventions portant loi uniforme», Clunet 1956, p. 570 ; H. Bauer, «Les traités et les règles de droit international privé matériel », Rev. crit. DIP 1966, p. 537.
} 
Certaines contraintes tiennent à la technique de codification, d'autres touchent au contenu du Code envisagé.

\section{$1 \%$ Les contraintes tenant à la technique de codification}

20. Le code peut être défini comme un ensemble de règles juridiques mises en forme, la codification constituant cette opération de mise en forme de règles en un ensemble ${ }^{1}$. Le choix est habituellement offert au législateur d'une codification compilation et d'une codification modification ${ }^{2}$. La spécificité de la matière à codifier commanderait ici une codification modification ${ }^{3}$. Il convient de tenir compte, en effet, de l'importante dimension jurisprudentielle de la matière. Codifier cette dernière supposerait la rédaction de solutions non écrites et autres principes généraux...De plus, l'insertion dans un code de règles jurisprudentielles modifierait la nature de ces dernières : «inscrire dans la loi une règle qui était auparavant d'origine purement jurisprudentielle, c'est en modifier l'autorité juridique en déplaçant la frontière entre ce qui relève du choix du Parlement et ce qui appartient au pouvoir d'interprétation du juge ${ }^{4}$.

\section{$2 \%$ Les contraintes tenant au contenu du Code}

21. Une première question se pose : la matière doit-elle être éclatée entre plusieurs Codes ou doit-elle être réunie en un seul ? La question est débattue de l'opportunité d'une codification globale et autonome, ou d'une insertion des règles de droit international privé dans les codes de chacune des matières concernées. Nous partageons l'opinion selon laquelle «seule l'élaboration d'un texte indépendant paraît répondre à l'idée de systématisation inhérente à l'idée de codification » 5 .

22. Une deuxième question doit être résolue, en préalable à toute entreprise de codification. Convient-il de comprendre, dans le périmètre codifié, tout le droit international privé, selon la conception française ${ }^{6}$ ou faut-il limiter l'entreprise à certaines de ses branches seulement? La question doit être posée, car il conviendra sans doute de tenir compte de la répartition législative et réglementaire des

\footnotetext{
${ }^{1}$ R. Cabrillac, op. cit., p. 63

${ }^{2}$ Sur ces deux manières de codifier, v. J.-L. Sourioux, « Codification et autres formes de systématisation du droit à l'époque actuelle. Le droit français », Journées de la Société de législation comparée, 1988, RID comp. 1989, numéro spécial, p. 145.

${ }^{3}$ R. Cabrillac, op. cit., p. 266 : «...une codification des principes généraux applicables à une matière semble également soulever d'importantes objections. La place des principes généraux dans la hiérarchie des normes n'étant pas déterminée avec précision, cette codification pourrait se heurter pour certains d'entre eux à l'argument, déjà évoqué pour les normes supranationales du nécessaire respect de cette hiérarchie. A cette objection générale valable pour toute codification s'ajoute une objection propre aux codifications-compilation: les principes généraux demeurent souvent non écrits, et leur codification supposerait leur rédaction, incompatible avec la réalisation d'une simple compilation. »

${ }^{4}$ Y. Robineau, « A propos des limites d'une codification à droit constant », AJDA, 1997, p. 657.

${ }^{5}$ D. Bureau, op. cit., $\mathrm{n}^{\circ} 49$.

${ }^{6}$ Conception qui regroupe dans cette matière la nationalité, la condition des étrangers, les conflits de lois et les conflits de juridiction.
} 
différentes branches, s'opposant sans doute à une fusion en un seul corpus, de l'ensemble ${ }^{1}$.

23. Le contenu du Code réfléchira sans doute le problème inhérent à la diversification des sources, à l'éclatement du droit international privé entre producteurs nationaux et internationaux de normes. Sans aller jusqu'à inclure (de quelle autorité ?) les normes de source internationale dans le code interne, ce dernier pourrait néanmoins marquer un lien avec elles : la codification interne pourrait ainsi prévoir expressément la réserve générale des conventions internationales ${ }^{2}$. Ce serait là la stricte application du principe de hiérarchie des normes. Le code pourrait, sinon, renvoyer aux dispositions d'une convention internationale. Le procédé demeure cependant peu envisageable en présence de traités aux multiples dispositions s'imbriquant dans le droit interne. Enfin, une présentation des normes internationales pourrait trouver sa place dans une simple annexe de chaque grande subdivision du Code : si ce parti était pris, il finirait par être difficile de distinguer une codification officielle d'une codification privée. Il semble bien impossible d'intégrer réellement et harmonieusement les sources internationales du droit international dans le corpus. Cela jouera certes, mais seulement sur le périmètre de la codification qui ne sera pas exhaustive, un point c'est tout ${ }^{3}$. Le dogme de l'exhaustivité de la codification n'a pas valeur absolue ni impérative ${ }^{4}$

24. Le développement des sources conventionnelles du droit international privé n'interdit pas une codification nationale qui pourrait au moins, ce ne serait pas rien, remédier à la crise des sources internes du droit. Cette codification nationale pourrait, d'ailleurs, progresser au côté d'une «codification ${ }^{5}$ internationale ${ }^{6}$. La préservation de la souplesse et de l'adaptabilité de la matière commande enfin une autre exigence, quant au contenu du code, celle de ne pas trop enserrer la jurisprudence pour permettre à celle-ci de cultiver une interprétation évolutive des dispositions. Ce code, répondant sur ce point au vœu de Portalis, doit fixer les grandes maximes et ne point trop entrer dans les détails ${ }^{7}$. A ces conditions, une codification est possible et pourrait être jugée souhaitable. Il demeure néanmoins à

\footnotetext{
${ }^{1}$ Imposant, au moins, une partie législative et une autre réglementaire.

${ }^{2}$ R. Cabrillac, op. cit., p. 264.

${ }^{3}$ En, ce sens, D. Bureau, op. cit., $\mathrm{n}^{\circ} 35$.

${ }^{4}$ R. Cabrillac, op. cit., p. 265.

${ }^{5} \mathrm{~V}$. supra, $\mathrm{n}^{\circ} 7$, les raisons des guillemets.

${ }^{6}$ En ce sens, R. Cabrillac, op. cit. ; contra, B. Oppetit, «Essai sur la codification », in Ethique et droit, PUF, p. 68: «Ainsi demeure incompréhensible, à ce jour, le double mouvement simultané de codification du droit international privé qui s'est développé à la fois dans le cadre national (dans plusieurs pays de grande tradition juridique) et à l'échelle internationale par la voie du droit conventionnel : comment un même pays peut-il s'engager en même temps dans deux processus distincts pour régir pourtant les mêmes questions ?»

7 «L'office de la loi est de fixer, par de grandes vues, les maximes générales du droit ; d'établir des principes féconds en conséquences, et non de descendre dans le détail des questions qui peuvent naître sur chaque matière »: Discours préliminaire sur le projet de code civil présenté le $1^{\text {er }}$ pluviôse an IX. Sur lequel v. Le discours et le Code. Portalis, deux siècles après le Code Napoléon, Litec, 2004.
} 
se prononcer sur le point de savoir si elle a quelque chance, dans un proche voire lointain avenir, de voir le jour.

\section{II/ LA PROBABILITE D’UNE CODIFICATION DU DROIT INTERNATIONAL PRIVE}

Certains éléments encouragent à penser qu'une prochaine codification du droit international privé est envisageable. D'autres, plus nombreux, font pourtant douter de cette issue.

\section{A/ Les éléments favorables à la codification}

25. Rémy Cabrillac ${ }^{1}$ a montré que l'alchimie de deux éléments est indispensable à l'éclosion de toute codification : la rencontre d'un besoin social de sécurité juridique et d'une forte volonté politique de codifier ${ }^{2}$. Le besoin social de sécurité juridique, s'il n'était pas flagrant en 1804 voire en 1950, aujourd'hui, existe. L'état actuel du développement des relations privées internationales l'engendre. Qui dit codification dit simplification, certitude, modernisation du droit ${ }^{3}$. La codification, codification, au minimum, en faciliterait la connaissance. Le souci est partagé par beaucoup de rendre le droit international privé plus simple et accessible ${ }^{4}$. L'entreprise pourrait, de manière heureuse, être entreprise conjointement à l'échelle nationale comme internationale.

26. La perspective d'une codification pourrait, substantiellement, s'imposer, la matière étant en proie aux incertitudes et imprévisibilités de la méthode conflictuelle, allant jusqu'à engendrer une importante critique de la méthode ellemême, dont on conteste aujourd'hui la suprématie pour le règlement des rapports internationaux ${ }^{5}$. Il y a, derrière cette contestation de la méthode, une critique non dissimulée de ses sources. Dans la plupart des pays, les règles de conflit ont une origine jurisprudentielle, non légale. Ceci engendre incertitude, imprévisibilité en raison, notamment, des fluctuations dont ces règles sont l'objet. La solution positive semble souvent découler non d'une règle bien assise, mais du sentiment contingent d'équité qu'entretient le juge, si ce n'est des exigences de l'opportunité. La théorie des conflits de lois, si elle est un jeu pour des savants intellectuels, ce qui n'est pas

\footnotetext{
${ }^{1}$ Les codifications, préc. p. 86.

${ }^{2}$ Sur ce dernier point, v. également B. Oppetit, « De la codification », Dalloz, Thèmes et commentaires, 1996, p. 7, spéc. p. 8

${ }^{3}$ Rousseau plaidait pour des « codes clairs, courts et précis » antidote de « ces foules de lois qui souvent se contredisent, dont le nombre rend les procès éternels et dont le conflit rend également les jugements arbitraires », in Considérations sur le Gouvernement de Pologne et sur sa réformation projetée, œuvres complètes, La Pléïade, tome 3, p. 951 et s., spéc. p. 1001.

${ }^{4}$ v. L. Gannagé, La hiérarchie des normes et les méthodes du droit international privé, Etude de droit international privé de la famille, LGDJ, Bibl. dr. privé, tome 353, préf. Y. Lequette, $\mathrm{n}^{\circ} 4$. L'exigence d'unité et de sécurité du droit serait, selon certains, inhérente au concept même de code. V. J. Vanderlinden, Le concept de code en Europe occidentale du XIIIe au XIXe siècles, Essai de définition, Bruxelles, 1967, p. 74 à 76.

${ }^{5}$ Sur cette question, v. Y. Loussouarn et P. Bourel, op. cit., $\mathrm{n}^{\circ} 64$ et s. ; B. Audit, « Flux et reflux de la crise des conflits de lois », Trav. Com. fr. DIP, Journée du cinquantenaire, 1989, p. 59.
} 
grave, risque d'être un procédé arbitraire entre les mains des juges, ce qui est infiniment plus dangereux. La codification pourrait constituer un moyen de juguler l'incertitude et l'imprévisibilité, donc de prévenir l'arbitraire ${ }^{1}$.

27. Certains verront encore d'autres atouts dans l'accessibilité plus aisée au droit que permettrait la codification du droit international privé, qui constituerait probablement un moyen de multiplier et diversifier les destinataires de la norme, d'éviter une certaine confiscation de la matière par une élite doctrinale, bien peu encline, selon certains, à partager le monopole qu'elle détient. La codification pourrait, encore, s'analyser en un frein au droit conventionnel. Il est admis que « les pays qui viennent d'introduire une nouvelle loi de droit international privé seront tenté d'insister sur les solutions qu'ils viennent de consacrer et de ne pas vouloir les abandonner par des solutions conventionnelles $»^{2}$. La codification pourrait ainsi accentuer le particularisme national du droit international privé et rendrait plus difficile l'acceptation de conventions internationales, ralentirait le mouvement d'unification. Le phénomène fut sensible au XIXe siècle, époque à laquelle les codifications ont eu pour effet d'engendrer une diversification des règles de droit international privé, diversification contrastant singulièrement avec la relative unité régnant en Europe au siècle précédant.

Un tel frein peut, à l'évidence, séduire en France, tandis que se multiplient les dénonciations des ravages du droit conventionnel. Néanmoins, une telle attitude n'est pas unanime et ne provient que d'une fraction de la doctrine, nouveau témoignage de ce que le droit international privé est le lieu de toutes les controverses, des oppositions les plus franches. Cet élément affecte lourdement la perspective d'une codification.

\section{B/ Les éléments défavorables à la codification}

28. Au regard des deux éléments dont la combinaison est indispensable à l'éclosion d'un code, si le besoin social existe aujourd'hui, on peut en revanche fortement douter de la volonté politique de codifier le droit international privé. Quel serait, il est vrai, le profit politique immédiat d'une telle entreprise ?

29. Sans doute faut-il, de plus, pour précipiter la codification d'une branche du droit, outre les deux éléments précédemment définis, une certaine unanimité doctrinale. Or, la doctrine de droit international privé est totalement éclatée, partagée par des courants, traversée par des opinions inconciliables. L'opposition est ainsi radicale entre les universalistes, convaincus de la nécessité de parvenir à une unification du droit international privé et affirmant, en conséquence, la primauté des sources internationales, et les particularistes, qui nient la possibilité même d'une unification de la matière. Nationalistes, ils préfèrent dès lors les sources internes aux sources internationales.

\footnotetext{
${ }^{1}$ Y. Loussouarn et P. Bourel, Droit international privé, Dalloz, $7^{\mathrm{e}}$ éd., p. 53.

${ }^{2}$ D. Bureau, op. cit., $\mathrm{n}^{\circ} 45$. C'est bien pourquoi l'Ecole historique allemande fut si hostile à la codification, au début du XIXe siècle.
} 
30. La perspective d'une codification interne ne pourrait que réjouir les particularistes. Cela conférerait un surplus d'autorité à la norme interne, d'une part, et constituerait un frein à l'unification, d'autre part ${ }^{1}$. Mais cette perspective hérisse les universalistes, qui pourraient dénoncer l'entrave à l'unification. Seule, pour ces derniers, une codification au niveau international aurait quelque sens, mais l'idée ne peut que heurter les autres. On a pu dire que ce qui avait fait la force et garanti la pérennité du Code civil est qu'il avait constitué un «extraordinaire compromis »². Le compromis semble, ici, introuvable.

31. Il faut aussi, peut-être, composer avec la crainte de la doctrine de se voir confisquer une part de son pouvoir d'influence ${ }^{3}$. La doctrine internationaliste fut prompte à faire l'éloge de la jurisprudence, à se féliciter de la remarquable qualité de l'œuvre normative de la jurisprudence française de droit international privé qui s'expliquerait, notamment, par le fait que l'élaboration des règles jurisprudentielles s'opère selon un processus de maturation des principes et des solutions dans lequel le dialogue avec la doctrine joue un rôle que lui refuserait la mécanique législative. Il y a ainsi fort à craindre la combinaison néfaste, pour la codification, d'une désaffection du politique et d'une résistance de la doctrine.

32. Au-delà, et fondamentalement, les destinataires de la norme, les praticiens, souhaitent-ils vraiment ce code ? Ne faut-il constater que derrière la prétendue crise des conflits de lois, sourd « une crise à la fois plus générale et plus profonde : celle du droit étatique auquel certains usagers aspirent à substituer le droit matériel d'un milieu donné, international par nature qui ne saurait s'identifier à un milieu étatique ni s'accommoder de dispositions du droit interne ? ${ }^{4} \mathrm{Ne}$ voit-on se dessiner les fondements d'une société internationale des commerçants, qui s'appuie sur des organisations professionnelles responsables de l'élaboration d'un droit corporatif, recourent à l'arbitrage... La codification serait une idée condamnée, non réellement compte tenu de ce qui fait son essence, mais compte tenu de son origine, étatique ${ }^{5}$.

33. L'avenir, en droit international privé, ne serait-il pas, dès lors, dans une codification privée ? «Une codification officielle imposée par un Etat ne constitue

\footnotetext{
${ }^{1}$ v. supra, $\mathrm{n}^{\circ} 27$

${ }^{2}$ R. Cabrillac, «L'avenir du Code civil», JCP 2004, I, 121, $\mathrm{n}^{\circ} 8$ : « Le Code civil constitue un extraordinaire compromis, compromis politique entre les différents courants de pensée de l'Ancien Régime et de la Révolution, compromis technique entre pays de droit écrit et pays de droit coutumier, sans doute inspiré par le profond esprit de modération de Montesquieu pour qui «l'esprit de modération doit être celui du législateur: le bien politique comme le bien moral, se trouve toujours entre deux limites » ».

${ }^{3}$ Pour un exemple particulier, et éclatant: Y. Lequette, «L'influence de l'œuvre d'Henri Battifol sur la jurisprudence française », Trav. Com. fr. DIP, 1991-1992, p. 32.

${ }^{4}$ Y. Loussarn et P. Bourel, préc., n ${ }^{\circ} 77$, p. 64 ; adde. E. Gaillard, Trente ans de lex mercatoria, JDI 1995, p. 5; Goldman, «La lex mercatoria dans les contrats et l'arbitrage internationaux: réalités et perspectives », Clunet 1979, p. 475 ; F. Osman, Les principes généraux de la lex mercatoria, contribution à l'étude d'un ordre juridique international, Paris 1992.

${ }^{5}$ Le propos pourrait valoir au moins pour le droit commercial international
} 
peut-être plus le modèle univoque qui s'impose $»^{1}$. Les difficultés des codificateurs contemporains, tiraillés entre le principe d'exhaustivité assigné à la codification et l'impossibilité d'appréhender dans la structure des codes les normes internationales, européennes ou non étatiques, témoignent de la nécessité de ce dépassement. Les codifications privées peuvent utilement relayer nombre de codifications officielles. La supériorité de ces publications privées, plus facilement évolutives, plus complètes puisqu'elles peuvent inclure les normes internationales, infra étatiques ou jurisprudentielles, apparaît sensible. Pour le droit international privé, elle est flagrante.

${ }^{1}$ R. Cabrillac, Les codifications, préc., p. 305. 
